\title{
Publisher Correction: Single-cell RNA-seq analysis software providers scramble to offer solutions
}

Michael Eisenstein

Correction to: Nature Biotechnology https://doi.org/10.1038/s41587-020-0449-8, published online 9 March 2020.

In the version of this article initially published, the development of dropSeqPipe was credited to the lab of high-throughput scRNA-seq pioneer Steve McCarroll. It should have been credited to Patrick Roelli and colleagues at the Swiss Institute of Bioinformatics. The error has been corrected in the HTML and PDF versions of the article.

Published online: 15 April 2020

https://doi.org/10.1038/s41587-020-0510-7

(C) The Author(s), under exclusive licence to Springer Nature America, Inc. 2020 